\title{
Discussion on Ways to Strengthen Construction Supervision of Road and Bridge
}

\author{
Liang Zhang \\ Daqing Petroleum Engineering Supervision Limited Company, Daqing 163000, China \\ $57578521 @ q q . c o m$
}

\begin{abstract}
The rapid development of China's economy in the past 30 years is inseparable from the perfect road and bridge transportation system, the completion of roads and bridges promote the economic development and cultural exchange of two places, it can be said that the construction of roads and bridges boost the rise of China's development. Therefore, it is crucial to make roads and bridges fully play their role and guarantee their quality. Under this circumstance, it is necessary to pay special attention to the supervision work in the construction process, the Hong Kong-ZhuhaiMacao Bridge that has just been built in China, if the supervision work cannot be done well, it is very likely that quality problems will occur; it does not play the purpose of communication between the two places. Therefore, it is of great significance to strengthen the discussion on the road construction supervision of roads and bridges, in this paper, this paper put forward some opinions on the characteristics of road and bridge construction and the problems in the supervision process, and hope to provide some reference for relevant staff.
\end{abstract}

Keywords: construction supervision of road and bridge; life and property; safety control.

\section{Introduction}

In the history of road and bridge construction in China, the occurrence of safety accidents is not uncommon, which has a certain impact on the completion schedule and cost of the overall project, and it has also attracted the attention of the current construction team. How to ensure the construction quality, avoid safety accidents, and prolong the service life of road bridges, there are problems that need to be considered by the construction side, in this case, the construction party must not only arrange the construction procedures reasonably, do good in the supervision work in the construction process, and the overall construction quality and economic benefits are guaranteed.

\section{The Effect of Supervision Work in the Road and Bridge Construction}

As far as the current social background is concerned, the supervision work in the construction process of roads and bridges has become a crucial link, which is related to the long-term development of the whole construction project, as for its root, the project supervision department is responsible to the proprietor, and the owner is its client. If the supervision department wants to play its own functions, it needs to rely on the supervision contract or the engineering contract, only when there is authorization, it can enter the project site for supervision. In the process of carrying out supervision work, it is difficult to effectively complete by the supervision department alone, it needs to communicate with the construction party and cooperate with each other, and achieve precise control of the relevant factors in each construction stage, strengthen the energy efficiency of supervision, all the safety indicators in the construction process of roads and bridges have been effectively improved, and guarantee that the construction efficiency reaches the expected target, and the construction risks are reasonably avoided to minimize the occurrence of safety accidents. Therefore, the supervision department needs to strictly control the safety in the actual work process, and avoid the occurrence of safety accidents under the condition of ensuring the quality of the project.

Scale is an inevitable trend in the construction of roads and bridges at this stage, under this trend, various uncertain factors are increasing in the construction phase, as an important component, project supervisor should clear its own obligation and shoulder its own responsibilities, the owner's needs as the goal and guidance of their own work, comprehensively consider the various refinement factors in the construction process of the road and bridge according to the relevant regulations, strictly supervise 
the construction quality according to the established standards, and ensure the construction quality cannot fail under any circumstances. Therefore, the energy efficiency control carried out during the supervision process has a direct impact on the subsequent supervision level, more importantly; it is also closely related to the quality of the project, if the supervision work can be completed efficiently, then the overall project quality will be improved; if the efficiency of the supervision work is relatively low, then it is impossible to accurately control the key factors involved in the project, which further leads to the continuous decline of construction quality, therefore, the supervision department must pre-set goals before the actual work, if the constant direction is missing, it is difficult to effectively control the work focus, which will lead to the formalization problem of supervision work.

\section{Characteristics of Road and Bridge Construction and Key Points of Supervision Work}

In the construction process of roads and bridges, the projects involved are very extensive, and the technology also has high requirements, and the weather changes can easily affect the actual construction of roads and bridges. In the actual construction process, it mainly has the following characteristics. The first is that the required working time is longer, in the general construction, it takes a few years, and even more than ten years, and some construction sites are relatively remote, which brings a big impact on the construction of construction workers. Secondly, the complex construction organization, in the process of road and bridge construction, it involves engineering mechanics, water conservancy engineering and civil engineering, this requires the supervisory staff to have relevant professional knowledge and rich work experience. At the same time, due to the oneness of road and bridge construction, this requires the construction unit to strictly implement the relevant laws and regulations formulated by the state; the supervision department must strictly follow the relevant regulations in the actual work process.

At present, China's economy is developing rapidly, road and bridge construction projects are gradually increasing, and the problems in the actual construction process are also different, the supervision department needs to carry out targeted work on these issues. First, the configuration facilities are not perfect, in some construction sites, due to various reasons; it cannot provide sufficient construction equipment, which causes the construction project cannot to be completed efficiently, there may be certain safety hazards and increase construction costs. Second, there are problems with project planning and surveying, after the overall construction is completed, it is necessary to carry out the later maintenance and management, if there is a problem here, it is easy to cause cracks in the bridge and seriously affect the safety of the road and bridge [1]. Moreover, some construction units have weak safety awareness and the quality of the staff is uneven, various construction items are piled up in the construction site, and the order is chaotic, which is easy to cause safety accidents. All these problems require the supervision department to pay more attention to it, seize the key points, and effectively supervise.

\section{Strengthen Supervision Methods of Road and Bridge Project}

\subsection{Ways to Strengthen Supervision in the Preparation Stage of the Project}

(1) In the preparation stage of the preliminary construction, all the materials involved in the construction project need to be strictly screened and reviewed, whether it is construction technology or construction equipment, it must be importantly measured to ensure that all relevant elements in the construction process can achieve expected results, and then guarantees the stability and safety of the whole construction project. (2) The various measuring equipment used in the construction process also need to verify its precision to ensure the accuracy of the actual measurement, after the inspection is correct, it can be put into the actual construction, and avoid the measurement error affect the quality of the whole project [2]. (3) The supervisor must focus on controlling all the applied materials in the construction process, strictly control the entry safety of materials, and prevent unqualified materials from appearing on the construction site, especially some important construction materials, which need 
to be checked many times. (4) Measure the construction quality strictly according to the standard, the supervisors must have rich professional knowledge and can effectively deal with the sudden problems in the measurement process; the staff must have the qualification certificate and use it as the first threshold. After the measurement is completed, it needs to be carefully compared with the actual standard, when it is found that there is an error, it is necessary to check and confirm the error again, when it is confirmed that it is not in conformity with the standard data, it can be fed back to the construction side, the measurement is performed after the rectification and reform is completed.

\subsection{Ways to Strengthen Supervision During the Implementation Phase of the Project}

(1) All raw materials that need to be used in the construction process must be examined and screened, after signed and confirmed by multiple supervisors, the raw materials can be sent to the construction site for use, once the quality reaches the standard or inferior materials, the entire batch of materials must be checked immediately, and the unqualified materials should be scrapped, especially those important materials, in the examination process, the construction technology should be taken into account, and only when it can meet the coordination requirements and can be put into the construction site for use. (2) In the measurement of construction process, the supervision department should regularly measure the measurement equipment to ensure that errors occur in measurement equipment during the long-term use, especially when sudden events occur or the construction plans change, the supervision unit needs to track and monitor the progress of material changes in real time, ensure that it can meet the requirements of the next measurement work, ensure the smooth and efficient development of subsequent supervision work, and reduce the occurrence of error problems. (3) During the development process of supervision work, the supervision department should grasp the change condition of the project in real time, so as to adjust the supervision work in time and update the construction plan, this requires the supervision department to ensure effective communication with the construction department, grasp the latest construction plan in time., and avoid using the previous supervision standards to affect the construction quality of the whole project, once there is something inconsistent with the newly updated construction plan, and require them to rectify immediately.

\subsection{Ways of Supervision in the Completion Stage of the Project}

After the construction of the road and bridge is completed, the supervision department is required to check the construction quality of the construction site, in this process, it is necessary to strictly follow the refinement standards and the construction design to measure good and bad points of the whole project, sign for those qualified parts, as for those parts whose quality is not up to standard, it is necessary to notify the constructor and handle it, after the rectification of the unqualified part is completed, it also needs to be checked again, and it can be signed after ensure that it meet the preset quality standards [4]. After these are completed, the supervision department is required to sort out all kinds of information and data generated during the construction phase, effectively combine it with the completed materials, comprehensively evaluate the quality of the whole project, and sign it after the qualification is confirmed. When carrying out this part of the work, the supervision department should comprehensively consider the problems in the construction and the characteristics of the project, and summarize it into own work experience in case of the subsequent work.

\section{Conclusion}

It can be seen from the above that it is of great significance to explore ways to carry out road and bridge supervision work, all construction workers should fully recognize the important effect of road and bridge construction supervisor in the whole project, such as improving comprehensive benefits, ensuring project quality, and adapting to the development trend of the times and so on. Moreover, because road and bridge construction have complex construction, organization and coordination, and long construction period, we should have a definite object in the process of carrying out supervision work. 


\section{References}

[1]. Ni Jun. Research and Discussion on Measures to Improve the Site Supervision Quality of Road and Bridge Project[J]. Sichuan Cement, 2015 (11): 00098-00098.

[2]. Hu Yang. Thoughts on Measures to Improve the Quality of Site Supervision of Road and Bridge Project[J]. Engineering Technology: 2016 (6): 00022-00022.

[3]. Zhang Chuanlun. Research and Discussion on How to Strengthen the Supervision of Road and Bridge Construction [J]. Engineering Technology: Full Text Edition, 2017 (2): 00219-00219.

[4]. Zhang Shuang, Zhang Yupeng. Brief Discussion on How to Improve the Quality of Site Supervision of Road and Bridge Engineering[J]. Sichuan Cement, 2017 (8): 36-36. 